\title{
Are Absence and Limbic Seizures Mutually Exclusive?: An Experimental Approach to Enigmatic Clinical Concept
}

\author{
Filiz Yılmaz Onat ${ }^{1,2}$ Esat Eşkazan ${ }^{3}$ \\ ${ }^{1}$ Department of Medical Pharmacology, School of Medicine, \\ Acıbadem Mehmet Ali Aydınlar University, Istanbul, Turkey \\ ${ }^{2}$ Department of Medical Pharmacology, Faculty of Medicine, \\ Marmara University, Istanbul, Turkey \\ ${ }^{3}$ Department of Pharmacology, Cerrahpaşa School of Medicine, \\ Istanbul, Turkey \\ J Pediatr Epilepsy 2021;10:45-50.
}

\begin{abstract}
Keywords

- limbic seizures

- absence seizures

- clinical concepts
\end{abstract}

\author{
Address for correspondence Filiz Yilmaz Onat, Department of \\ Medical Pharmacology, School of Medicine, Acıbadem Mehmet Ali \\ Aydınlar University, Istanbul 34684, Turkey \\ (e-mail: fonatmarmara@gmail.com; fonat@marmara.edu.tr).
}

\section{Introduction}

Despite the fact that generalized versus focal epilepsy on clinical side has been accepted as an enigma, no satisfactory clarifying explanation has yet been identified. ${ }^{1-5}$ Although improvements in video-electroencephalography (EEG), functional magnetic resonance imaging, magnetic resonance spectroscopy, positron emission tomography, super-resolution microscopy, sequencing-based gene, or variant discovery approaches greatly increased our understanding in enigmatic concepts, primary generalized, and focal onset in seizures or the interactions between the various elements of the systems in epilepsies have been a long-debated matter. ${ }^{6-8}$ Here, we tested the hypothesis that whether the mechanisms underly-

received

December 1, 2020 accepted

December 1, 2020

published online

February 2, 2021 ing focal limbic epilepsy are distinctively diverse than those responsible for genetic generalized epilepsies (previously known as idiopathic generalized) by using a combination of electrophysiological, genetic, and pharmacological models in rats. The overall outcome so far is actually translating to learning a lot about the phenomenology of the coexistence of absence epilepsy and limbic focal-onset in the same patient. ${ }^{9}$

\section{Brief Overview to Focal and Generalized Epilepsy Models: Phenotypic, Electroencephalographic, and Pharmacological Features}

Several animal models have been developed since the initial identification of phenytoin in 1937 by Merritt and Putnam by (c) 2021. Thieme. All rights reserved. Georg Thieme Verlag KG, Rüdigerstraße 14, 70469 Stuttgart, Germany
DOI https://doi.org/ 10.1055/s-0041-1722870. ISSN 2146-457X.
Epilepsy-An Enigma; Guest Editors: Mohammed llyas, MD, and Uğur Işık, MD 
using the cat maximal electroshock (MES) seizure test. ${ }^{10}$ As a general rule, an animal model should be reproducible, predictable, quantifiable, amenable to standardization, and consistent with bioethics of experimentation. ${ }^{11}$ To be a valid investigative tool, an animal model of generalized or focal epilepsies should reflect the phenotypic, EEG, and pharmacological characteristics of these disorders. ${ }^{12,13}$ There are several experimental in vitro and in vivo models of generalized and focal epilepsy induced by chemical and electrophysiological interventions and genetic manipulations. These models partially or fully meet the criteria and reflect the phenotypic, EEG, and pharmacological features seen in human epilepsy. We are aware that no model is likely to be perfect when animals are employed for a human condition. Instead, the initial model should be chosen on the basis of "best fit" for the situation. ${ }^{14,15}$ A well-validated animal models in well-defined experimental designs certainly provides a value to clarify the debatable clinical issues.

It has long been accepted that absence epilepsy as a form of genetic (idiopathic) generalized epilepsy serves as a model for an approach to generalized epilepsies In this context, typical absence epilepsy as the prototype of genetic (idiopathic) generalized epilepsies is characterized by several daily episodes of absence seizures with intermittent impairment of consciousness or brief interruption (arrest) of behavioral activity and by spontaneous spike-and-wave discharge (SWD) paroxysms, each consisting of high-voltage spike and subsequent slow wave components in the EEG. ${ }^{13}$ Typical nonconvulsive seizures of absence epilepsy are considered to be generated by a rhythmogenic interplay between the cortex and the thalamus and related to a predominance of reticulo-thalamic inhibitory activity and cortical hyperexcitability.

Experimental animal models of absence seizures can be divided into pharmacological or genetic strategy. ${ }^{11}$ Pharmacological strategy to induce absence seizures are triggered by some chemical substances in healthy animals. ${ }^{12}$ The genetic models include naturally occurring mutations in rats (Genetic Absence Epilepsy Rats from Strasbourg [GAERS] and Wistar Albino Glaxo rats from Rijswijk [WAG/Rij]) and in mice single locus mutation and transgenic models. GAERS and WAG/Rij rats are both well validated and commonly used genetic models of absence epilepsy. ${ }^{13,16-19}$ In these models, the SWDs, which are indeed a hallmark of absence epilepsy, are generalized high-amplitude asymmetric, synchronized, and rhythmic cortical activity expressed as SWD complexes lasting at least 1 second with a fundamental frequency of 7 to $11 \mathrm{~Hz}$ and with the first and second harmonics in spectrograms. ${ }^{20}$ Although the frequency of this phenomenon in humans $(\geq 2.5 \mathrm{~Hz})$ differs from the frequency in these models, SWDs and absence seizures share many similar electrophysiological, pharmacological, and behavioral characteristics in human absence epilepsy. 3,18

Focal epilepsies accounting for $60 \%$ of all forms of epilepsy traditionally have been regarded as largely acquired disorders. $^{21,22}$ This perception is related to the common observation that the epilepsy resulting from an environmental insultsuch as a stroke, infection, head trauma, or tumor-is focal. In many patients with focal epilepsy, there is a nongenetic cause for seizures such as febrile convulsions, head trauma, stroke, or brain infection. This initial insult triggers a cascade of neurobiological events during a latent period corresponding to epileptogenesis. $^{23,24}$ A cascade of neurobiological events following the initial insult includes both rapid and slower progressive changes, leading to excitotoxicity, inflammation, gliosis, neuronal loss, neurogenesis, plasticity, angiogenesis, molecular reorganization, and others. In experimental models of focal epilepsy, one of the most widely used and popular methodological tools is kindling. This historical method, kindling, addresses focal seizure development with secondary generalization due to its progressive manner and additionally resembles the progressive psychiatric and neurological changes accompanying epilepsy. The initial nonconvulsive stimulus induces a permanent seizure susceptibility and lasting brain alterations that are similar to that found in human temporal lobe epilepsy. ${ }^{25}$ Temporal lobe epilepsy covers a variety of disorders that have the common feature of seizures that arise in the temporal lobe. ${ }^{4,8,9}$

A phenomenon of kindling was first described in 1967 by Goddard and then became a major research tool for studying seizures involving the limbic system. ${ }^{26}$ Kindling as an animal model of temporal lobe or limbic epilepsy is usually defined as a progressive, cumulative, and permanent increase in seizure activity with repeated-usually once or twice daily -application of a stimulus. ${ }^{27-29}$ In this model, chemical or electrical stimulation of a single site in the brain induces an after-discharge in the EEG and behavioral changes. ${ }^{29}$ With repeated focal stimulations for days or weeks, there is a gradual increase in the duration of the after-discharge and behavioral seizure development until the animal's behavior progresses to the point of a full convulsions. ${ }^{30,31}$ In chemical kindling, repeated exposure to subconvulsive doses of a proconvulsant agent leads to increase in seizure activity, resulting in generalized seizures. The chemical kindling is usually triggered by the repetitive administrations of subconvulsive doses of pentylenetetrazole $(25-45 \mathrm{mg} / \mathrm{kg}$, s.c.). Both electrical and chemical-induced kindling are the most commonly used models for the understanding of limbic epilepsy and epileptogenesis.

\section{Are Absence Epilepsy and Temporal Lobe Epilepsy Mutually Exclusive to Each Other?}

To address a mutual cross-interaction between absence epilepsy and mesial temporal lobe/limbic epilepsy in GAERS and WAG/Rij rats as absence epilepsy models, electrical kindling and intra-amygdaloid kainic acid were used as the models of temporal lobe epilepsy. Our first clue regarding possible interactions between the cortico-thalamo-cortical and the limbic circuitry arose from the kindling experiments. Kindling studies by Eskazan et al, Onat et al, and Aker et al showed that when the animals are stimulated at their afterdischarge thresholds, the transition from stage 2 to 3 is absent or delayed in GAERS and WAG/Rij models., $9,32,33$ Although Wistar animals all reached stage 5 with repeated electrical amygdaloid kindling stimulations, GAERS and one group of WAG/Rij rats stayed at stage 2 after 30 stimulations that was the maximum number used. ${ }^{32}$ GAERS and WAG/Rij 
demonstrated higher after discharge thresholds than the Wistar rats suggesting that genetically absence epilepsy rats were less susceptible than nonepileptic animals. In addition, the genetic absence epilepsy rats (both GAERS and WAG/Rij groups), having the highest number of SWDs on the first day of the experiment, before the stimulation, were the least susceptible to kindling; in other words, a high level of SWD activity in the absence epilepsy rats reduces the progress of kindling. ${ }^{33}$ Spearmen correlation analysis showed a negative correlation between absence seizures and kindling rate in rats with genetic absence epilepsy. Further, hippocampal kindling resembles amygdaloid kindling in showing a delay of or resistance to secondary seizure generalization. ${ }^{34}$ In agreement with the clinical observations in human, experimental studies of temporal lobe epilepsy in rats with genetic absence epilepsy show a resistance to, or a delay of, secondary generalization of focal limbic seizures in the amygdaloid and hippocampal kindling experiments. Moreover, we investigated the hypothesis that whether there is an age-related difference in the resistance to secondary generalization to amygdaloid kindling. ${ }^{35}$ At 20 days of postnatal, when no SWDs are recordable in GAERS, all the animals were kindling prone. At 30 days of postnatal, when the first SWDs are detected, less than half of the GAERS (41\%) were resistant to secondary generalization (kindling resistant) and at 60 days of postnatal, when SWDs are consistently present, the percent of kindling-resistant GAERS was $69 \%$. In fact, all adult GAERS were kindling resistant. These results demonstrate that resistance to the secondary generalization of limbic seizures during amygdaloid kindling in GAERS increases with age as the SWDs on the EEG and possibly mechanisms underlying absence epilepsy mature. Further, GAERS during the development of the kindling course at stage 2 has demonstrated increased blood flow in the somatosensory cortex, ventrobasal, and mediodorsal thalamus as well as the entorhinal cortex, amygdala, hippocampus, nucleus accumbens, and substantia nigra pars reticulata. These results suggest a large widespread functional activation with kindling stimulations in GAERS and a relationship between thalamo-cortical and limbic structures in absence epilepsy.

The experimental approach started with a focus on absence versus limbic seizure phenomenology and took a major step forward when rats with genetic absence epilepsy (GAERS and WAG/Rij) show a reduced capacity for the production of experimental temporal lobe epilepsy by kindling. ${ }^{36}$ In considering the circuitry of a focal kindling seizure, there are at least three stages in the evolution of a seizure that may involve different circuit components: transition from interictal to ictal phase, the early phase of activity that occurs within the initiating circuit and seizure spread. Seizure spread covers the process of recruiting additional regions and circuits. ${ }^{36}$ The importance of breaking seizures down into these phases is that each is likely associated with different circuitry that involves different physiologies. ${ }^{36}$

To maximize the reliability of the data collected and analyzed in our laboratory, we further evaluated the interplay between absence epilepsy and limbic epilepsy by using intra- amygdaloid injections of kainic acid as another model of temporal lobe epilepsy to compare with the kindling findings in adult GAERS. ${ }^{37}$ In total, 1 week after the implantation of the recording electrodes and a cannula through the stereotaxic surgery, the animals were given a single injection of kainic acid (750 ng) into the basolateral amygdala of GAERS and Wistar rats. The EEG and behavioral changes after the kainic acid injection were evaluated over a 3-month period. At the end of the 3-month period, sections of the hippocampus were stained by the Neo- Timm method. In the acute phase, an amplitude increase in the gamma range $(25-45 \mathrm{~Hz})$ of spectrograms was observed within the first 40 minutes after the kainic acid injection in both the Wistar and the GAERS groups, and in the Wistar rats the amplitude increase remained almost constant for the rest of the 3-hour period. The activity in this frequency range remained at a lower level in the GAERS group and did not show any further increase even after the start of convulsive seizures at 100 minutes indicating the delay of the convulsive seizures in genetic absence epilepsy rats. Medvedev et al reported that an injection of kainic acid increases the gamma activity in rats. ${ }^{38}$ In vivo and in vitro studies suggest that gamma activity is produced by glutamatergic cells in rat hippocampus and neocortex. ${ }^{39}$ The gamma oscillations after the kainic acid injection reflect the level of synchronization in the cortex, which probably is one of the important determinants of the occurrence of convulsive seizures, and this synchronization is less marked in GAERS than in Wistar rats provided electrophysiological and morphological clues to improve our understanding on the enigmatic issue of the rare coexistence of absence epilepsy and limbic focal-onset seizures.

In the chronic period, the first convulsive seizure on the day of the kainic acid injection and spontaneous convulsive seizures over the 3-month period were significantly delayed in GAERS compared with control animals. ${ }^{37}$ Thereafter, the animals in both groups became epileptic and there was no difference in the survival rates of Wistar and GAERS. Thus, additional evidence for a mutual cross-inhibition of the circuits involved in limbic versus generalized absence epilepsy is demonstrated by our kainic acid administration findings. These include particularly the delayed convulsive seizures in the short-term during kainic acid-induced status epilepticus and long-term periods and the reduced sprouting of mossy fibers in GAERS. Additionally, the loss and reappearance of the SWDs, the changes occurring in the spikeand-wave discharges. These results are in accordance with previous findings by Vergnes et al. ${ }^{40}$ They showed that the first seizure induced by low kainic acid doses ( $5 \mathrm{mg} / \mathrm{kg}$, i.p.) was less severe and had a longer latency in GAERS than in nonepileptic controls. ${ }^{40}$

Furthermore, a digenic mouse model of absence epilepsy and temporal lobe epilepsy by combining two epilepsyassociated ion channel mutations in the same mice reveals a protective interaction. ${ }^{41}$ Increasing membrane excitability by removing Shaker-like $\mathrm{K}+$ channels, which are encoded by the Kcna1 gene, masked the absence epilepsy caused by a $\mathrm{P} / \mathrm{Q}$-type $\mathrm{Ca}^{2+}$ channelopathy due to a missense mutation in the Cacna1a gene, whereas decreasing network excitability 
by impairing Cacna1a Ca2 +-channel function reduced the occurrence of limbic seizures and sudden death in Kcna1 null mice. Nanobashvili et al reported that co-stimulation of the thalamic reticular nucleus during hippocampal kindling stimulation reduced the number and the duration of generalized convulsions. ${ }^{42}$ They concluded that thalamic reticular nucleus stimulations suppressed limbic motor seizures in hippocampal kindling and provided a new approach for seizure control in temporal lobe epilepsy. Altogether, these findings point to interactions between cortico-thalamo-cortical and limbic circuitry but do not show how and where the interactions occur.

Another issue covers the effect of temporal lobe epilepsy on absence seizures. When the kindling-resistant postnatal 30-day-old and postnatal 60-day-old GAERS were compared with the kindling-prone group, the kindling resistant animals showed a significantly longer duration of baseline SWDs on the first day of the experiments before the kindling stimulations. ${ }^{43,44}$ In addition, the kindling-prone GAERS showed an almost complete loss of SWDs after the first stage 5 in the pre-stimulation and post-stimulation periods. Further, in another temporal lobe epilepsy model, the kainic acid model, intensive basal SWD activity in GAERS was completely suppressed immediately after a kainic acid injection and then gradually reappeared over a period of 2 months. ${ }^{43,44}$ The disappearance of SWDs seen at the kindling stage 5 seizures or after the kainic acid injections suggests that the limbic seizures spread to areas involved in absence epilepsy mechanisms and modify their activity. In accordance with these findings, electrical co-stimulation of the thalamic reticular nucleus during the rapid hippocampal kindling procedure suppresses the development of generalized limbic seizures in the adult control Wistar rats. The outcomes in experimental temporal lobe epilepsy in GAERS, WAG/Rij, and Wistar rats have made another step toward a better understanding of the interaction between the limbic circuitry and cortico-thalamo-cortical network.

These findings all point to interactions between corticothalamo-cortical and limbic circuitry and further indicate a mutual cross-inhibition of circuits underlying absence epilepsy and limbic epilepsy in which seizures often start from the mesial temporal lobe. However, they do not show how these interactions occur. As one possibility, the thalamic reticular nucleus, which is involved in the expression and generation of SWDs in absence epilepsy, may prove to be a critical component in producing the resistance to the generalization of limbic seizures. This could occur through the connections of the reticular nucleus with the amygdala and hippocampus, which involve two-way connections between the reticular nucleus and thalamic nuclei close to the midline such as the mediodorsal, centromedial, reuniens, and anterior nuclei. ${ }^{45}$ The connections of the rostral thalamic reticular nucleus with limbic structures through mediodorsal, reuniens, centromedial, and anterior thalamic nuclei may be particularly relevant for understanding the resistance to secondary generalized convulsive seizures in the genetic absence epilepsy models or the kindling-induced decreases in SWDs (-Fig. 1). In vivo single neuronal recordings demonstrate that the evolution to

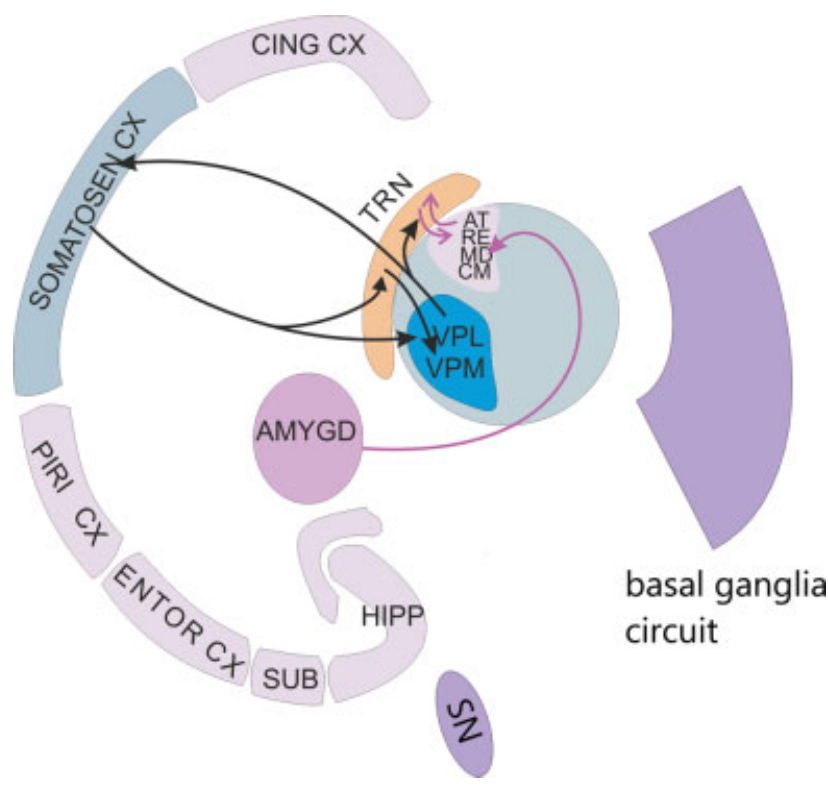

Fig. 1 Schematic illustration showing the interactions between cortico-thalamo-cortical and limbic circuitries. The cortico-thalamocortical network can be activated through the propagation of kindling seizures outside the amygdala to the limbic thalamic nuclei (anterior thalamic, reuniens, mediodorsal thalamic, and centromedial thalamic nucleus) and cortex. The amygdaloid nuclei are connected with the limbic thalamic nuclei, which have two-way links to the rostral part of the thalamic reticular nucleus, limbic reticular nucleus. In addition to the cortico-thalamo-cortical system in the pathophysiology of absence epilepsy network, the involvement of basal ganglia has also been demonstrated in childhood absence epilepsy and experimental absences. ${ }^{47,48}$ Hipp, hippocampus; Sub, subthalamic nucleus; TRN, thalamic reticular nucleus; VPM, ventroposteromedial thalamic nucleus; VPL, ventroposterolateral thalamic nucleus. The figure has been adapted and modified from Carcak et al (2009) with permission from Wiley.

generalized motor seizures in nonepileptic Wistar rats is associated with a change in interictal firing in the thalamic reticular nucleus to an epileptiform, burst firing pattern, and early engagement of neurons of this nucleus during the seizures. ${ }^{45}$ These findings are absence in rats with genetic absence epilepsy (GAERS). ${ }^{45}$ The same number of amygdala stimulations failed to induce the changes in interictal neuronal firing patterns in thalamic reticular nucleus neurons in GAERS. Kindling findings in GAERS have made a first step toward an understanding of the interaction between the limbic circuitry and cortico-thalamo-cortical network.

\section{Conclusion}

It has been hypothesized that the mechanisms underlying focal epilepsy are distinctively diverse than those responsible for generalized epilepsies. Studying the dissimilarities in focal and generalized epilepsies in experimental animal models to be representative of the human condition enable us the advantage of exploring the underlying mechanisms of these disorders. The experimental approach to the rare coexistence of limbic focal epilepsy and absence epilepsy as representative of genetic generalized epilepsies points to the mutual cross inhibition of circuitries involved in these 
two particular pathophysiological conditions. This approach also provides a clue for the underlying neurobiological mechanism of the clinical rare coexistence of absence epilepsy and mesial temporal lobe epilepsy in the same patient. Meaningful results emerge from animal testing when the preclinical strategy is part of a well-developed translational plan rather than a single experiment, often relying on clinical observations to guide preclinical development. ${ }^{46}$

\section{Funding}

None.

\section{Conflict of Interest}

None declared.

\section{References}

1 Geller EB, Lancman ME, Van Ness PC, et al. Coexistence of generalized and partial epilepsies. Electroencephalogr Clin Neurophysiol 1995;2(95):17P

2 Sofue A, Okumura A, Negoro T, et al. Absence seizures in patients with localization-related epilepsy. Brain Dev 2003;25(06): 422-426

3 Lüttjohann A, van Luijtelaar G. Dynamics of networks during absence seizure's on- and offset in rodents and man. Front Physiol 2015;6:16

4 Koutroumanidis M, Hennessy MJ, Elwes RD, Binnie CD, Polkey CE. Coexistence of temporal lobe and idiopathic generalized epilepsies. Neurology 1999;53(03):490-495

5 Nicolson A, Chadwick DW, Smith DF. The coexistence of idiopathic generalized epilepsy and partial epilepsy. Epilepsia 2004;45(06): 682-685

6 Jeha LE, Morris HH, Burgess RC. Coexistence of focal and idiopathic generalized epilepsy in the same patient population. Seizure 2006;15(01):28-34

7 Whelan CD, Altmann A, Botía JA, et al. Structural brain abnormalities in the common epilepsies assessed in a worldwide ENIGMA study. Brain 2018;141(02):391-408

8 Italiano D, Ferlazzo E, Gasparini S, et al. Generalized versus partial reflex seizures: a review. Seizure 2014;23(07):512-520

9 Aker RG, Yananli HR, Gurbanova AA, et al. Amygdala kindling in the WAG/Rij rat model of absence epilepsy. Epilepsia 2006;47 (01):33-40

10 Putnam TJ, Merritt HH. Experimental determination of the anticonvulsant properties of some phenyl derivatives. Science 1937 85(2213):525-526

11 Kostopoulos GK. Pharmacologically Induced Animal Models of Absence Seizures. In: Pitkanen A, Buckmaster PS, Galanopoulou AS, Moshé SL, eds. Models of Seizures and Epilepsy. USA: Elsevier Inc, Academic Press; 2017:553-567

12 Cortez MA, Snead OC. Pharmacologic models of generalized absence seizures in rodents. In: Pitkanen A, Schwartzkroin PA, Moshé SL, eds. Models of Seizures and Epilepsy. USA: Elsevier Inc, Academic Press; 2006:111-126

13 Onat FY, van Luijtelaar G, Nehlig A, Snead OC III. The involvement of limbic structures in typical and atypical absence epilepsy. Epilepsy Res 2013;103(2-3):111-123

14 Löscher W. Fit for purpose application of currently existing animal models in the discovery of novel epilepsy therapies. Epilepsy Res 2016;126:157-184

15 Barker-Haliski M, SteveWhite H. Validated animal models for antiseizure drug (ASD) discovery: advantages and potential pitfalls in ASD screening. Neuropharmacology 2020:167

16 Coenen AM, Van Luijtelaar EL. The WAG/Rij rat model for absence epilepsy: age and sex factors. Epilepsy Res 1987;1(05):297-301
17 Cortez MA, Perez Velazquez JL, Snead OC III. Animal models of epilepsy and progressive effects of seizures. Adv Neurol 2006; 97:293-304

18 Crunelli V, Leresche N. Childhood absence epilepsy: genes, channels, neurons and networks. Nat Rev Neurosci 2002;3(05): 371-382

19 Danober L, Deransart C, Depaulis A, Vergnes M, Marescaux C. Pathophysiological mechanisms of genetic absence epilepsy in the rat. Prog Neurobiol 1998;55(01):27-57

20 Akman O, Demiralp T, Ateş N, Onat FY. Electroencephalographic differences between WAG/Rij and GAERS rat models of absence epilepsy. Epilepsy Res 2010;89(2-3):185-193

21 Bertram EH. Temporal lobe epilepsy: where do the seizures really begin? Epilepsy Behav 2009;14(1, Suppl 1):32-37

22 Perucca P. Genetics of focal epilepsies: what do we know and where are we heading? Epilepsy Curr 2018;18(06):356-362

23 Sloviter RS, Bumanglag AV. Defining "epileptogenesis" and identifying "antiepileptogenic targets" in animal models of acquired temporal lobe epilepsy is not as simple as it might seem. Neuropharmacology 2013;69:3-15

24 Engel J Jr. Mesial temporal lobe epilepsy: what have we learned? Neuroscientist 2001;7(04):340-352

25 Sato M, Racine RJ, McIntyre DC. Kindling: basic mechanisms and clinical validity. Electroencephalogr Clin Neurophysiol 1990;76 (05):459-472

26 Goddard GV. Development of epileptic seizures through brain stimulation at low intensity. Nature 1967;214(5092):1020-1021

27 McIntyre DC, Racine RJ. Kindling mechanisms: current progress on an experimental epilepsy model. Prog Neurobiol 1986;27(01): 1-12

28 Bertram E. The relevance of kindling for human epilepsy. Epilepsia 2007;48(Suppl 2):65-74

29 McIntyre DC, Gilby KL. Kindling as a model of human epilepsy. Can J Neurol Sci 2009;36(Suppl 2):S33-S35

30 Sutula T, Cascino G, Cavazos J, Parada I, Ramirez L. Mossy fiber synaptic reorganization in the epileptic human temporal lobe. Ann Neurol 1989;26(03):321-330

31 Cavazos JE, Golarai G, Sutula TP. Mossy fiber synaptic reorganization induced by kindling: time course of development, progression, and permanence. J Neurosci 1991;11(09):2795-2803

32 Eşkazan E, Onat FY, Aker R, Oner G. Resistance to propagation of amygdaloid kindling seizures in rats with genetic absence epilepsy. Epilepsia 2002;43(10):1115-1119

33 Onat FY, Eşkazan E, Aker R. Experimental absence versus amygdaloid kindling. Kindling 6 Boston, MA: Springer; 37-472005

34 Akman O, Karson A, Aker RG, Ates N, Onat FY. Hippocampal kindling in rats with absence epilepsy resembles amygdaloid kindling. Epilepsy Res 2008;81(2-3):211-219

35 Carçak N, Aker RG, Özdemir O, Demiralp T, Onat FY. The relationship between age-related development of spike-and-wave discharges and the resistance to amygdaloid kindling in rats with genetic absence epilepsy. Neurobiol Dis 2008;32(03):355-363

36 Bertram EH. Neuronal circuits in epilepsy: do they matter? Exp Neurol 2013;244:67-74

37 Gurbanova AA, Aker RG, Sirvanci S, Demiralp T, Onat FY. Intraamygdaloid injection of kainic acid in rats with genetic absence epilepsy: the relationship of typical absence epilepsy and temporal lobe epilepsy. J Neurosci 2008;28(31):7828-7836

38 Medvedev A, Mackenzie L, Hiscock JJ, Willoughby JO. Kainic acid induces distinct types of epileptiform discharge with differential involvement of hippocampus and neocortex. Brain Res Bull 2000; 52(02):89-98

39 Herrmann CS, Demiralp T. Human EEG gamma oscillations in neuropsychiatric disorders. Clin Neurophysiol 2005;116(12): 2719-2733

40 Vergnes M, Boehrer A, Reibel S, Simler S, Marescaux C. Selective susceptibility to inhibitors of GABA synthesis and antagonists of 
$\operatorname{GABA}(\mathrm{A})$ receptor in rats with genetic absence epilepsy. Exp Neurol 2000;161(02):714-723

41 Glasscock E, Qian J, Yoo JW, Noebels JL. Masking epilepsy by combining two epilepsy genes. Nat Neurosci 2007;10(12):1554-1558

42 Nanobashvili Z, Chachua T, Nanobashvili A, Bilanishvili I, Lindvall O, Kokaia Z. Suppression of limbic motor seizures by electrical stimulation in thalamic reticular nucleus. Exp Neurol 2003;181(02):224-230

43 Onat FY, Aker RG, Gurbanova AA, Ateş N, van Luijtelaar G. The effect of generalized absence seizures on the progression of kindling in the rat. Epilepsia 2007;48(Suppl 5):150-156

44 Carçak N, Ferrandon A, Koning E, et al. Effect of stage 2 kindling on local cerebral blood flow rates in rats with genetic absence epilepsy. Epilepsia 2009;50(01):33-43
45 Carçak N, Zheng T, Ali I, et al. The effect of amygdala kindling on neuronal firing patterns in the lateral thalamus in the GAERS model of absence epilepsy. Epilepsia 2014;55(05):654-665

46 Barker-Haliski M, Friedman D, White HS, French JA. How clinical development can, and should, inform translational science. Neuron 2014;84(03):582-593

47 Paz JT, Chavez M, Saillet S, Deniau JM, Charpier S. Activity of ventral medial thalamic neurons during absence seizures and modulation of cortical paroxysms by the nigrothalamic pathway. J Neurosci 2007;27(04):929-941

48 Moeller F, Siebner HR, Wolff S, et al. Changes in activity of striatothalamo-cortical network precede generalized spike wave discharges. Neuroimage 2008;39(04):1839-1849 